\title{
EFFICIENCY OF 6- AND 12-PUNCTURES BIOPSIES TO DETECT PROSTATE CANCER IN PATIENTS WITH PSA $\leq 10$ NG/ML AND NORMAL DIGITAL RECTAL EXAMINATION
}

\author{
LUIZ E. SLONGO, MÁRIO C. SUGISAWA, SÉRGIO O. IOSHII, RENATO TÂMBARA FILHO, \\ LUIZ C.A. ROCHA \\ Division of Urology, School of Medicine, Federal University of Paraná, Curitiba, Paraná, Brazil
}

\begin{abstract}
Objective: Establish the efficiency of 6- and 12-punctures transrectal ultrasound-guided needle biopsies in low risk patients for prostate cancer. Six-punctures (sextant) biopsies were compared to 12-punctures biopsies, assessing which is the best strategy to detect this neoplasm.

Materials and Methods: Among 240 patients submitted to prostate biopsy, 54 with suspected small and organ-localized tumors (prostatic specific antigen $\leq 10 \mathrm{ng} / \mathrm{mL}$ and digital exam of the prostate not suggesting cancer) in glands $<50 \mathrm{~cm}^{3}$ were selected, constituting a homogenous sample. These patients were submitted to standard 3-punctures (basal, mid, and apical) sextant biopsy in parasagittal midline of each prostatic lobe, with 3 additional lateral punctures, bilaterally. Each specimen was separately submitted to histological study.

Results: Twenty-two (40.7\%) patients had prostatic cancer, and 28 presented prostatic hyperplasia, associated or not to inflammatory conditions. High-grade prostatic intraepithelial neoplasia (PIN) was detected in 4 patients. From 22 tumors detected by 12-punctures biopsies, 6-punctures biopsies in the parasagittal midline (sextant) diagnosed $50 \%$ of the cases, while isolated lateral punctures diagnosed $90.9 \%$ of the malignant neoplasms. Basal lateral punctures responded for $72.7 \%$ of the cancer diagnosis, while basal sextant punctures responded only for $9.1 \%$ of the cases.

Conclusion: For low risk prostate cancer, patients' 12-punctures biopsy was more effective, for sextant biopsy failed to diagnose half of the cases of neoplasm. Three lateral punctures (basal, mid, and apical), with 2 additional punctures in the parasagittal midline (mid and apical) bilaterally are suggested as the best biopsy strategy.
\end{abstract}

Key words: prostate; prostatic neoplasms; biopsy; diagnosis; needle

Int Braz J Urol. 2003; 29: 24-9

\section{INTRODUCTION}

The prostatic cancer $(\mathrm{PCa})$ is the most diagnosed male sex neoplasia in Occident, supplanted in mortality only by lung malignancies. The PCa diagnosis is confirmed by the histological evaluation of fragments obtained by prostate gland biopsy.

The transrectal ultrasound-guided needle prostate biopsy, directed to parasagittal midline of both prostatic lobes, in basal, mid and apical portions, with the addition of punctures directed to ultrasonographic suspect lesions (designated as sextant biopsy), has been used as standard since the end of the eighties. This technique was first described by Hodge et al. in 1989, for patients presenting digital rectal exam and/or prostatic ultrasonographic study with suspicion of malignancy (1). Since the 90's, with the widespread use of prostatic specific antigen (PSA) measurement as screening to early detection of $\mathrm{PCa}$, a new challenge was elicited to confirm this diagnostic suspicion, leading to new strategies of $\mathrm{BxP}$, mainly for patients with PSA dosage slightly elevated, and digital exam of the prostate not suggesting cancer. 
Following this new reality, the requirement to repeat sextant $\mathrm{BxP}$ for patients with persistently high measurements, without other diseases explaining such biochemical abnormality, was observed. A significant percentage of these patients, when submitted to the new biopsy, received PCa diagnosis, showing that sextant biopsy underestimated the presence of prostatic cancer $(2,3)$. Nevertheless, the false negative results of the sextant $\mathrm{BxP}$ presented in literature are inconsistent, suggesting that the patients' samples observed were not homogenous. Studies generally compared the efficiency to detect $\mathrm{PCa}$ in patients with high PSA measurements and/or digital exam of the prostate suspect for malignancy, with no other consideration. Therefore, patients presenting extensive prostatic tumors, which are easily sampled by just one puncture, were compared to patients presenting minimal disease, difficult to detect.

Keeping in mind that today BxP is frequently indicated to patients presenting only slight raises in PSA measures, this study was designed with the aim of establishing $\mathrm{PCa}$ incidence and ascertaining the efficiency of 12-punctures BxP compared to 6-punctures $\mathrm{BxP}$ in this population.

\section{MATERIALS AND METHODS}

This research was approved by the Committee for Ethics in Research with Humans of our University. Among 240 consecutive patients referred to prostatic biopsy by local urologists in the period of January $17^{\text {th }}, 2000$ to November $20^{\text {th }}, 2000$. Among these, 54 cases were selected fulfilling the following requirements: a) digital exam of the prostate not suggesting cancer; b) PSA measurement $\leq 10 \mathrm{ng} / \mathrm{mL}$; and c) prostatic gland $<50 \mathrm{~cm}^{3}$ by transrectal ultrasound.

All biopsies were performed by the same examiner. An ultrasound Siemens Sonoline Versa Plus ${ }^{\circledR}$ with transrectal transducer $6.5 \mathrm{MHz}$ biopsy automatic pistol with 18-gauge needles equipment was used. Antimicrobial prophylaxis was performed with oral ciprofloxacin $500 \mathrm{mg}$ q12 hours for 3 consecutive days, initiating 24 hours before the procedure. Bowel preparation consisted in cathartic enema 1 hour before the procedure.
To carry out the exam, the patients were placed in left lateral position, and sedation was performed with intravenous propofol. Before the biopsy, a new digital exam of the prostate was performed to confirm the gland features. Sequentially, a prostate ultrasonography was performed, establishing its volume, and the presence or absence of suspicious hypoechogenic or nodular images.

Twelve consistent prostate punctures were performed at: a)- the parasagittal midline of the prostate (sextant punctures). Right lobe: base, mid region, and apex (specimens 1,2, and 3, respectively); and Left lobe: base, mid region, and apex (specimens 4, 5 , and 6 respectively); b)- equidistant between the margin and the parasagittal midline: (lateral punctures). Right lobe: base, mid region, and apex (specimens 7, 8, and 9, respectively); and Left lobe: base, mid region, and apex, (specimens 10, 11, and 12, respectively) (Figures-1, 2, and 3).

An additional sample was taken when there was an ultrasonographic suspicion of neoplasm in an area, and this was included in the systematic sampling of the region. Nevertheless, for glands of greater volume with a hypertrophic transition zone constricting the peripheral zone, it was determined that the surgeon should increment the needle's angulation intending to removing a sample of the constricted peripheral zone and its anterior horn. Each specimen was fixed in a strip of filter paper, and its extremity was delimited with green Indian ink. Subsequently, it was separately packed in labeled vials containing $10 \%$ formalin solution, representing 12 samples. All cases were analyzed by the same pathologist, according to Prophet et al., and Bostwick \& Dundore $(4,5)$ criteria.

For statistic analysis the parametric Student's "t" test of and the non-parametric "Comparisons between 2 Proportions" and " $\chi$-square" for independent samples.

\section{RESULTS}

Mean age of selected patients in this study was $57.7 \pm 9.8$ years, ranging from 41 to 80 years. The average of total PSA was $6.5 \pm 1.7 \mathrm{ng} / \mathrm{mL}$, ranging from 2.7 to $10.0 \mathrm{ng} / \mathrm{mL}$. Three patients presented 


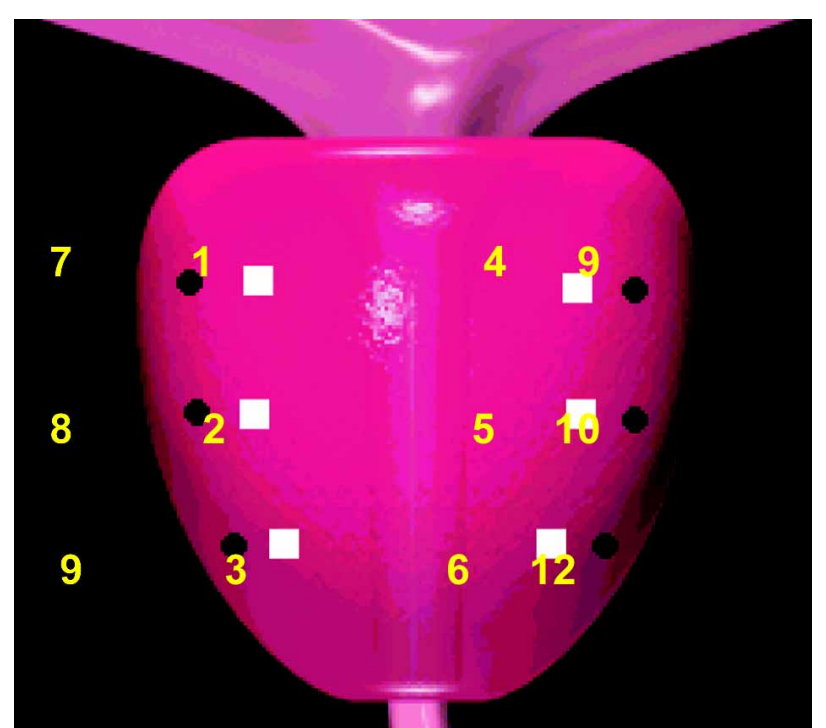

Figure 1 - Sextant punctures in white and lateral in black (authorized by Sanofi-Synthelabo).

PSA total measurement $=4 \mathrm{ng} / \mathrm{mL}$, of which 2 presented total PSA of $4 \mathrm{ng} / \mathrm{mL}$ and only 1 patient with 42 years presented total PSA of $2.7 \mathrm{ng} / \mathrm{mL}$. Prostatic mean volume by transrectal ultrasound was $34.7 \pm$ $8.3 \mathrm{~cm}^{3}$, the smallest gland presenting $17.0 \mathrm{~cm}^{3}$ and the biggest $49.0 \mathrm{~cm}^{3}$.

Amidst 54 patients selected and submitted to systematic prostate biopsy, $22(40.7 \%)$ cases were

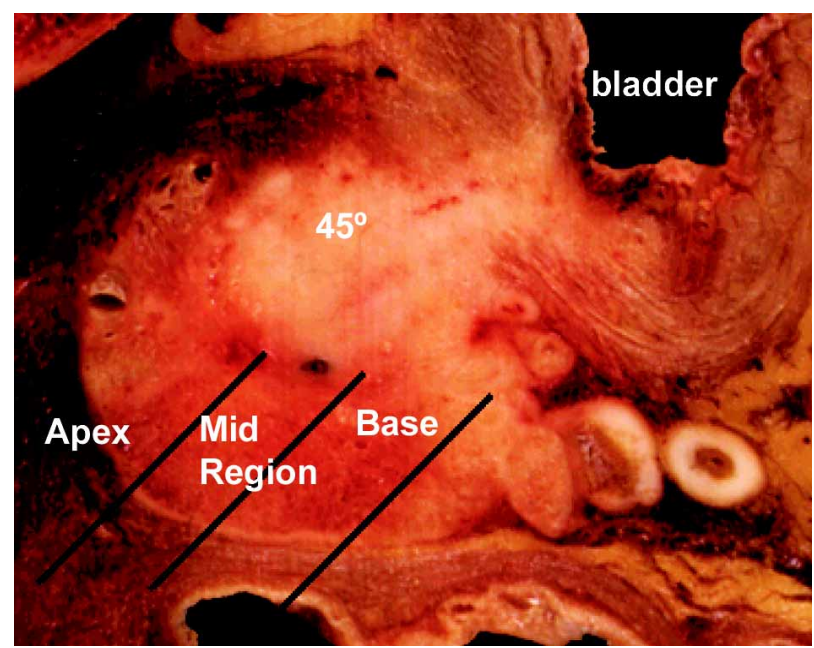

Figure 2 - Sagittal section of the prostate, showing the localization of punctures (authorized by Sanofi-Synthelabo). positive for cancer, and the mean Gleason score was 5.5, ranging from 4 to 8 . Among the remainder 32 patients, $20(62.5 \%)$ presented benign prostatic hyperplasia $(\mathrm{BPH})$ associated with chronic prostatitis, 7 (21.9\%) presented only BPH, $3(9.4 \%)$ presented $\mathrm{BPH}$, chronic prostatitis, and prostatic intra-epithelial neoplasia, 1 patient (3.1\%) presented BPH associated with acute and chronic prostatitis, and another (3.1\%) presented BPH associated with prostatic intra-epithelial neoplasia.

Among 22 patients with $\mathrm{PCa}$ diagnosis, 9 $(40.9 \%)$ cases had cancer detected by sextant punctures and lateral punctures as well. Individually, lateral punctures detected $\mathrm{PCa}$ in 11 cases $(50.0 \%)$, while sextant punctures detected only 2 cases $(9.1 \%)$ of malignant tumors. Therefore, sextant punctures were positive for PCa in $11(50.0 \%)$ cases, and lateral punctures in $20(90.9 \%)$ cases $(\mathrm{p}=0.008)$, primarily lateral basal $(72.7 \%)(\mathrm{p}=0.007)($ Table-1).

In the analysis of the biopsy technique performed compared to the total of patients studied, we have noticed that sextant punctures diagnosed $\mathrm{PCa}$ in $20.4 \%$ of the total of patients, whilst sextant + lateral punctures were diagnostic in $40.7 \%(\mathrm{p}=0.0367)$.

When the diagnostic percentage was analyzed according to the biopsy technique used, within the group of patients with established prostate can-

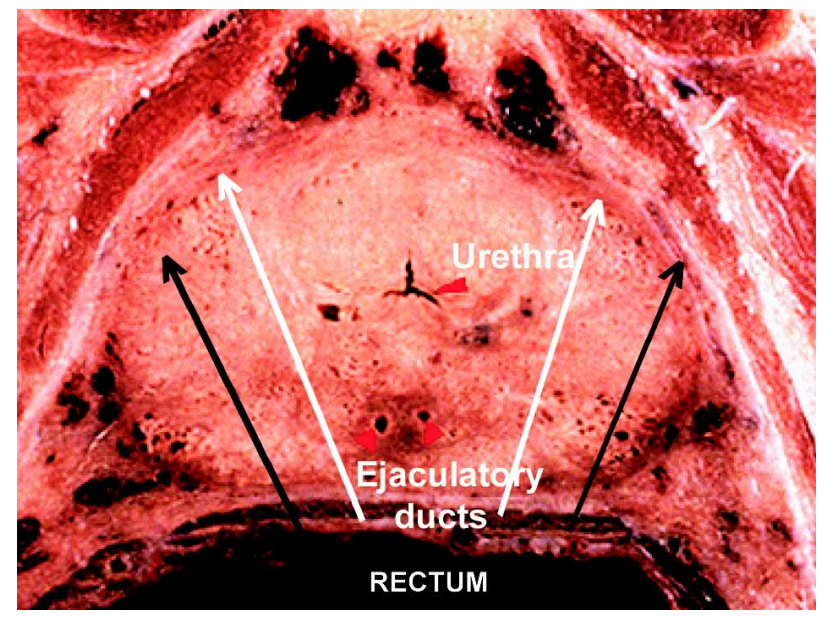

Figure 3 - Transverse section of the prostate, showing the localization of the punctures. Sextant punctures in white and lateral in black (authorized by Sanofi-Synthelabo). 
Table 1 - Regions of the positive punctures for cancer.

\begin{tabular}{lcc}
\hline $\begin{array}{c}\text { Positive Punctures } \\
\text { for Cancer }\end{array}$ & $\mathbf{N}^{\mathbf{0}}$ Patients & Percentage \\
\hline Superior sextant & 02 & 9.1 \\
Mid sextant & 08 & 36.4 \\
Inferior sextant & 10 & 45.5 \\
Superior peripheral lateral & 16 & 72.7 \\
Mid peripheral lateral & 10 & 45.5 \\
Inferior peripheral lateral & 11 & 50.0 \\
\hline
\end{tabular}

cer $(\mathrm{n}=22)$, sextant technique would fail to diagnose $50.0 \%$ of the patients with prostate cancer from the selected group ( $\mathrm{p}<0.0001)$, while sextant + lateral peripheral punctures diagnosed $100.0 \%$ of the cases.

\section{DISCUSSION}

Patients presenting a digital rectal exam not suggesting cancer, and persist with biochemical suspicion of malignancy due to high PSA measures, frequently require a second prostate biopsy.

Many of these patients, after confirming $\mathrm{PCa}$ with a second biopsy, are submitted to radical prostatectomy, and in the histological study of the surgical specimen frequently a neoplasm of significant volume is detected, with no reasonable explanation for the fact that the first biopsy could not detect the tumor. We observed that there is not a consensus in literature regarding the optimal technique for prostate biopsy. The percentage of detected tumors and false negative results are inconsistent owing to lack of stratification. The same technique approach was employed in all the cases without differentiating nonpalpable minimum disease from bulky tumors. $\mathrm{Pa}$ tients with PSA slightly elevated were compared to patients with very high PSA, and generally a biopsy strategy considering the volume of the prostatic gland was not observed. Therefore, cases with tumors of difficult detection by biopsy were compared to cases with tumors easily detected by only one puncture, in this way it is justified the false negative findings described in literature, ranging from $1 \%$ to $35 \%(6,7)$.
With the idea of establishing the efficacy in detection of PCa of the 12-punctures biopsies compared to sextant biopsies, a patient sample with similar characteristics and with low risk for PCa was selected. These patients presented PSA dosage $\leq 10 \mathrm{ng} /$ $\mathrm{mL}$, non-palpable tumors and glands $<50 \mathrm{~cm}^{3}(8)$. Excluding cases with large prostates $\left(\geq 50 \mathrm{~cm}^{3}\right)$ had the objective of evaluating the glands in which volume the PCa occurs with greater frequency (9), as well as using only one standard biopsy technique approach in order not to have much variation in prostates' volume. This sample of patients presenting similar features was considered ideal to compare between 12- and 6-punctures biopsies. All conventional technical resources were used to diagnose $\mathrm{PCa}$, yet punctures in the prostate midline were avoided as in this region only 2 a $4.1 \%$ of the tumors $(7,10)$ are observed, and these punctures account for higher morbidity of the procedure.

Among 240 systematically biopsied patients, 54 cases (with previously mentioned features) constituted an homogeneous sample of patients with clinic suspicion of small volume prostate tumors, which are difficult to detect by biopsy. Of 54 selected patients, 22 (40.9\%) presented PCa. Among the remainder 32 patients, $21(65.6 \%)$ presented acute and or chronic inflammatory conditions associated to BPH, 7 (21.9\%) only BPH, and 4 (12.5\%) high-grade intraepithelial neoplasia.

The detected tumors index in the present study was high, similar to the one found by Eskew et al. (7) in the so-called 5 regions biopsies, for patients with high PSA and/or palpable tumor. This must be highlighted, given that this population is clinically considered as presenting low risk for PCa. The diagnostic of high grade PIN was detected in $12.5 \%$ of the patients, which is an index similar to the one reported by Levine et al. (11). Nonetheless, Martins et al. detected high grade PIN in $7.3 \%$ of the Brazilian population above 40 years with PSA $>4 \mathrm{ng} / \mathrm{mL}$ and digital rectal exam suspicious for $\mathrm{PCa}$ (12).

From a total of 22 patients with prostatic cancer diagnosed by 12-punctures biopsy, $50 \%$ were detected by isolated lateral punctures - these tumors would not be diagnosed by the sextant biopsy, $(\mathrm{p}=0.008)-$ and $9,1 \%$ of the tumors were detected 
only by the sextant punctures. The remaining tumors $(40.9 \%)$ were detected by both sextant and lateral punctures. The sextant puncture, according to literature, fails to diagnose up to $35 \%$ of the $\mathrm{PCa}$ in patients with high PSA and/or suspicious digital rectal exam without other stratifications $(6,7,13)$. Nevertheless Beurton et al. (14) in a protocol with one sample from patients with similar features (stratified digital rectal exam and PSA data), reported that sextant biopsy failed to diagnose PCa in $47 \%$ of the cases.

Various publications confirmed the existence of false negative results from sextant biopsy; yet, the false negative index ranged according to the employed methodology $(6,13)$. Ballantine Carter (10) underlined that sextant technique was idealized in a time where diagnostic suspicion was entirely based in digital rectal exam and sonographic findings; that in PSA era malignant lesions usually are detected when small and still not palpable, and in this phase are found more laterally, and may not be detected by sextant biopsy. This was actually corroborated by Boboruglu et al. (13) report that documented the existence of PCa in $30 \%$ of the patients presenting previous negative sextant prostate biopsy and persisted with clinical suspicion of cancer.

On the other hand, Naughton et al. (6) evaluated 244 patients with PSA between 2.5 and $20 \mathrm{ng} / \mathrm{mL}$ and/or rectal exam suspicious for prostate cancer, detecting cancer in $26 \%$ and $27 \%$, respectively, by 6 - and 12-punctures biopsy. This was the only published study in which the greater number of punctures failed to yield a significant increase in the detection index of PCa. However, this protocol did not stratify the patients for digital exam of the prostate, comparing non-palpable tumors with extensive tumors.

The issue of the possibility of more than 6punctures biopsies identify an excessive number of non-significant tumors $(<0.5 \mathrm{cc}$ and Gleason score $\leq$ 4) was elucidated by many authors. Among them, Digiuseppe et al. (15) that found the presence of nonsignificant disease in merely $2.8 \%$ of 3.038 radical prostatectomies studied, in patients without previous hormonal treatment or prostate resection, showing a low incidence of PCa without clinical meaning.

In the present study, the anatomic region where the greater contribution for the cancer diag- nostic was obtained was the lateral regions in prostate base in $72.7 \%$ of the cases (punctures \#7 and 10), while the places where the presence of PCa was less frequently detected were the sextant regions, also in the base of the gland (punctures \#1 and 4), in 9.1\% of the cases. For none of the patients isolated basal sextant punctures detected neoplasia, so this punctures may be excluded from the protocol without harm. This data is in accord to the papers published by Presti Jr. $(16,17)$.

\section{CONCLUSION}

In the selected group of patients (PSA $\leq 10$ $\mathrm{ng} / \mathrm{mL}$, digital examination of the prostate not suggesting malignancy, and glands $<50 \mathrm{~cm}^{3}$ ), the incidence of prostate cancer was high. The 12-punctures biopsies were more efficient in detecting neoplasia compared to sextant biopsies. However, it is suggested as better strategy for prostate biopsy, for patients with this features; 3 lateral punctures (basal, mid and apex), added with 2 punctures in the parasagittal midline (mid and apex), bilaterally.

\section{REFERENCES}

1. Hodge KK, Mcneal JE, Terris MK, Stamey TA: Radon systematic versus directed ultrasound guided transrectal core biopsies of the prostate. J Urol. 1989;142:71-5.

2. Stamey TA: Making the most out of six systematic sextant biopsies. Urology. 1995; 45: 2-12.

3. Presti Jr JD, Chang JJ, Bhargava V, Shinohara K: The Optimal systematic prostate scheme should include 8 than 6 biopsies: Results of a prospective clinical trial. J Urol. 2000; 163: 163-7.

4. Prophet EB, Arrington JB, Sobin LH: Laboratory methods in histotecnology. Armed Forces Institute of Pathology. American Registry of Pathology. Washington, DC. USA, 1994.

5. Bostwick DG, Dundore PA: Biopsy pathology of the prostate. London UK, 1997.

6. Naughtton CK, Miller DC, Mager DE, Ornstein DK, Catalona WJ: A prospective randomized trial comparing 6 versus 12 prostate biopsy cores: Impact on cancer detection. J Urol. 2000; 164: 388-92 
7. Eskew LA, Bare RL, Mccullough DL: Systematic 5 region biopsy is superior to sextant method for diagnosing carcinoma of the prostate. J Urol. 1997; 157: 199-203.

8. Catalona WJ, Richie JP, Ahmann FR, Hudson MA, Scardino PT, Flanigan RC: Comparison of digital rectal examination and serum prostate specific antigen in the early detection of prostate cancer: Results of a multicenter clinical trial of 6.630 men. J Urol. 1994; 151: $1283-90$.

9. Uzzo RG, Wei JT, Waldbaum RS, Perlmutter AP, Byrne JC, Vaughan Jr. ED: The influence of prostate size on cancer detection. Urology. 1995; 46: 831-6.

10. Ballentine Carter H: Is sextant prostate biopsy the standard of the care in PSA era. AUA News. 1999; 4: 1-3.

11. Levine MA, Ittman M, Melamed J, Lepor H: Two consecutive sets of transrectal ultrasound guided sextant biopsies of the prostate for the detection of prostate cancer. J Urol. 1998; 159: 471-6.

12. Martins ACP, Reis RB, Suaid HJ, Maciel LMZ,
Cologna AJ, Falconi RAR: Screening for carcinoma of the prostate in volunteers. Braz J Urol. 2000; 26: 516-22.

13. Borboroglu PG, Comer SW, Riffenburgh RH, Amling CL: Extensive repeat transrectal ultrasound guided prostate biopsy in patients with previous benign sextant biopsies. J Urol. 2000; 163: 158-62.

14. Beurton D, Barthelemy Y, Fontaine E, Chartier E, Lamotte F, Franc B: 12 systematic proatate biopsies are superior to sextant biopsies for diagnosing carcinoma: A propective randomized study. Brit J Urol. 1997; 80 (Suppl 2): 239.

15. Digiuseppe JA, Sauvageot J, Epsein JI: Increasing incidence of minimal residual cancer in radical prostatectomy specimens. Am J Surg Pathol. 1997; 21: 174-8.

16. Presti Jr JD: How to biopsy the prostate. AUA News. 1999; 4: 27-30.

17. Presti Jr JD: Systematic biopsies of the prostate: 6 just aren't enough. Cont Urol. 1999 ; 11: 11-7.

Received: March, 262003

Accepted after revision: December, 182003

\author{
$\overline{\text { Correspondence address: }}$ \\ Dr. Luiz Edison Slongo \\ Rua Portugal, 329 \\ Curitiba, PR, 85510-280, Brazil \\ Fax: + $55413241-1329$ \\ E-mail: slongo.uro@mps.com.br
}

